\title{
Study on Innovative Model of E-learning Based on SNS under Background of Web 2.0
}

\author{
Kai Xiong \\ Business school, Jianghan University, Manufacturing Development \& Research Center of Wuhan city circle, Wuhan, Hubei, \\ China (xkyx@sina.com)
}

\begin{abstract}
Under background of Web2.0, the traditional mode of education is faced with severe challenges. E-learning has become a new business model, which is paid much attention by the majority of the education sector and investors. Due to its features of information sharing and interactive exchange, Social Networking Service (SNS) grows very quickly and its value and benefits are also increasingly prominent. As an application of the Web, SNS' features and functionality determine it has vigorous potential in the field of E-learning. From the angle of technical level and the application level, SNS is very suitable with E-learning. The innovative model of E-learning based on SNS can achieve its profit objectives from angles of resources' share, answers inquiries, various aspects discuss, popular electronic transactions, and forum lecturing.
\end{abstract}

Keywords - E-learning, SNS, innovation

\section{Web 2.0 背景下 E-learning 模式创新研究—-SNS 视角 \\ 熊凯}

江汉大学商学院, 武汉城市圈制造业发展研究中心, 武汉, 湖北, 中国

\begin{abstract}
摘 要 在 Web2.0 背景下, 传统的教育模式正受到严峻挑战, E-learning 逐渐成为一种新的商业模式, 受到广大教育界和投资 者的青睐。社会性网络服务 (SNS) 由于其信息分享性和交流互动性的特点, 发展速度和发展潜力都呈现蓬勃的上升态势, 其价值和优 势也日益凸显。作为 Web 的一种运用, SNS 的特点和功能决定了它在 E-learning 领域大有作为。从技术层面和应用层面来看, E-learning 与 SNS 的结合非常适宜。基于 SNS 的 E-learning 创新模式可以从资源共享、问询解答、热门讨论、电子交易、设坛开讲等多个方面实 现其盈利的目的。
\end{abstract}

关键词 E-learning, SNS, 创新

1. 引言

随着互联网从 Web1.0 时代进入到 Web 2.0 时代, 传统 的教育模式受到了严峻的挑战, E-learning 正逐渐成为一种 新的商业模式, 逐渐受到广大教育界及其投资者的大力关 注。为了挖掘并强占这个具有巨大利润空间的市场, 许多 商家都在教育行业寻求最具商业潜力的盈利模式。布庸置 疑, 一个成功的网络教育盈利模式将会给创业者和投资者 带来巨大的商机和财富。

SNS (social networking services), 也称社会性网络服 务, 是指帮助 Web2.0 时代的互联网人士构建社会性交互网

武汉市教育局 2013 年市属高校教学研究重点项目支持(2013021)
络的新型 web 服务。SNS 网络是根据真实社会关系和人际 关系而建设起来的虚拟网络社区, 利用 SNS 网络, 传统 E-learning 可以突破网络教育的时空, 建立网络学习新情景 和新模式, 使教师和学生之间的交流更加实时、开放、畅 通和有效, 形成完全开放化、民主化、自由化的符合建构 主义学习理论的新的学习环境。

\section{SNS 概述}

2.1 SNS 概念、特点及功能

在Web2.0 时代, SNS 有三层含义: 社会网络服务 (social network service)、社会网络软件（social network software）、 
社会网络网站（social network site）, 通常的理解是社会性 网络服务或社交网络服务, 是指为互联网人士构建构建社 会性交互网络的新型 web 服务。维基百科认为 SNS 是指集 中帮助那些分享兴趣与活动或者喜好探索他人兴趣与爱好 的人们建立的一种在线沟通交流平台, 并且这些网站提供 了诸如 e-mail、即时通讯等各种互动的方式。Boyd D.M 和 Ellison N.B (2007) 认为, SNS 允许个体有下面三种功能 一种基于网络的服务:（1）在一定的圈子或领域范围内营 造完全公开或者部分公开部分保密的个人资料信息;（2） 罗列与其他个体共享连接用户的一个 List; (3) 查看并且 遍历他们的链接列表和其他人在此系统内提出的列表。由 此可以看出, SNS 的本质在于人的互动性, 它允许人与人 之间信息的双向传播, 允许人与人之间相互以分享兴趣、 活动、经验的形式形成互动, 并通过 SNS 的这种互动性达 到广泛传播的效果。SNS 是基于 Web2.0 的理念提出来的, 它追求的是实现每个人的个人价值, 鼓励用户积极主动的 参与分享之间的生活与学习。

SNS 具有两个重要特点: 分享性和交流互动性。分享 性是指所有的用户都可以在这个平台上分享各自的兴趣、 爱好和经验; 互动性是指用户之间的交流是自由的、畅通 的和平等的。SNS 通过这两个特点来聚焦人气, 实现增强 用户粘性, 从而最终实现通过 SNS 提高个人的人脉价值。 从功能上来看, SNS 具有六大基本功能:（1）身份管理。 个体主要通过营建个性特征的电子资料信息数据库来管理 互联网身份及个人信息; (2) 寻找专家。研究表明通过寻 找专家可以更好地帮助个人外化隐形知识; (3) 情景感知。 情景感知是指不同个体对同一事物或情景的感知, 来自于 共同的关注人、爱好、同学或同事; (4) 联系管理。联系 管理将联合所有那些帮助人们维持与建立人与人数字化人 际网络的功能; (5) 网络意识。SNS 的功能支持在人际关 系网络中的联系人的活动意识, 允许人们通过这种活动意 识进行间接沟通;（6）交换。交换是指人们之间直接或间 接的分享信息，包括视频、音频、照片等。

\subsection{SNS 网站发展现状}

2003 年, Friendster.com 带动了美国 SNS 的第一个热 潮, 不到半年时间, Friendster 就拥有 400 万注册用户, 一 年后达到 800 万用户。2003 年, Friendster 获得了风险投资 商 Kleier Perkins 以及 Benchmark \& Battery 公司 1300 万美 元的风投。自此以后, 世界范围内的 SNS 蜂拥而至, 遍地 开花。与此同时, 中国也出现了许多类似 Friendster 网站, 如 UUme、YeeYoo、heiyou 等。但 SNS 在中国大规模的发 展直到 2008 年才真正开始。2008 年 6 月, 风靡 “办公室 一族” 的开心网在行业内引起巨大反响, 用户在这段时间
内发展迅速井喷, 发展速度令人瞠目结舌。同时, 热门网 站校内网的大股东千橡集团获得日本软银公司 3.4 亿美元 的风投, Face book 也从微软和李嘉诚基金获得巨大的融 资, 由此带来世界主要资本市场对 SNS 的投资达到了前所 未有的高度（古玉立、李大美，2008）[1]。

\subsection{SNS 网站优势分析}

SNS 网站的典型特征是拥有大量的用户群, 其关注度 和点击率都远远高于传统网站。而且, 由于要关注其他联 系人, 因而其可以起到很强的口碑传播效应。相比较于传 统的广告媒体, 这种口碑传播不仅成本比较低, 而且其传 播范围更广, 传播速度更快, 可信度更高。比如, 在 Face book 上的一篇博文, 经过博文主人的联系人, 以及联系人 的其他联系人, 可以把这篇博文扩散到更广泛的人群。而 且, 通过 SNS 网站来实施广告的精准率比一般网站的精准 率更高。据权威互联网研究报告称, 一般传统网站的用户 精准率不到 5\%, Google 的精准率为 12\%, 而实名制 SNS 网站精准率可达到 35\%以上。看见, SNS 在精准营销方面 具有传统网站不可比拟的优势。

SNS 网站具有巨大的商业价值。2007 年 10 月, 微软 斥资以 2.4 亿美元购买了 Facebook 不到 $2 \%$ 的股份, 这一 举动让业界看到了 SNS 未来巨大的商业潜力。2008 年, 校 内网的控股股东千橡集团总共得到了 4.3 亿美元的融资, 其中 3.9 亿美元来自于软银几天, 其他部分则来自于 Joho Capital 和 SBI 提供的首轮战略融资。业界认为, 校内网之 所以受到风险投资商如此高的青睐, 原因在于其在 SNS 领 域无与伦比的领先优势。

与传统的网站一样, SNS 网站的赢利模式也不外乎三 种: 网络广告、网站增值服务和电子商务 (丁喆, 2006) [2]。据统计, 2007 年, 美国网络社区广告费用投放达到 9.2 亿美元。美国一家权威互联网研究机构预计, 2014 年全世 界的社交网站广告毛收入将达到 70 亿美元。可见, 广告收 入仍然还是社交网站最核心的赢利方式。但相比于传统的 网站, SNS 网站由于其客户资源的质量比较优化, 在精准 营销方面具有强大优势, 可以为广告商们提供更加精准的 广告业务, 更容易受到广告业主的青睐（程绍珊、席加省, 2006）[3]。同时, SNS 还可以推出一些个性化的其他产品 服务, 如微博招聘、微博营销、同城推荐等服务, 使其其 优势更加明显和巨大。

\section{E-learning 传统模式分析}

E-learning 也被称作在线教育或网络教育, 是依靠互联 网技术而迅速普及的一种新型的教育模式, 它以信息技术 为支撑, 以建构主义学习理论为指导, 打破时空界限, 实 
现学习者自主学习、远程管理的一种教育模式 (万明义, 2006）[4]。与传统的教育模式相比, E-learning 具有实时性、 交互性、情景性、多样性等基本特征。

E-learning 的传统模式有三种: B2C、B2B、C2C（田 桂平, 2006）[5]。B2C 模式是目前网络培训学校最常用的 商业盈利模式。在这种模式中, 网校提供优质教学资源, 如教学资料、教学视频、教学答疑等服务内容, 以出售学 习卡或网上交易充值的方式将这些优质资源捆绑销售给学 习者。 $\mathrm{B} 2 \mathrm{C}$ 模式以学习者为主导, 学习者可以根据自己的 需要进行相应内容的学习, 并且可以反复学习直到完全掌 握为止。同时, 这种模式可以实现在线答疑, 网校可以解 决学习者在线学习遇到的各种问题, 为客户提供全方位的 服务。

B2B 模式是指网络学校借助于其品牌效应, 将其教学 资源先出售给其他培训机构或中小学校, 再由他们选择其 中的教学内容对学生进行辅助教学。这种模式的特点是网 络教育学校与其他培训机构或中小学校之间建立了合作联 盟, 双方形成了较稳固的合作关系, 利益共享。相对于 B2C 模式, 这种模式客户群更稳定, 利润空间更大。而且, 网 校可以为合作的培训机构或中小学校量身定制他们所需要 的网络课程或 CAI 课件, 双方的合作更有针对性和持久性。

$\mathrm{C} 2 \mathrm{C}$ 模式是指以 IM 即时通讯为技术环境, 通过 web 和即时通讯相结合, 为教师和学习者之间营造一个良好的 教育教学平台, 并且在这种平台上实现教学互动的一种教 学模式。 $\mathrm{C} 2 \mathrm{C}$ 模式的最大特点在于其可以实现网络教育的 定制化服务, 真正实现 “一个家庭教师” 为一个学生进行 “一对一” 的教学辅导。该种模式目前在 E-learning 中应 用还比较少, 主要针对一些特定的学习群体, 目前该种模 式已经开发出一些诸如心理健康服务、父母亲子交流活动 等多种丰富多彩的增值服务。

\section{E-learning 模式创新研究: SNS 视角}

\subsection{E-learning 与 SNS 的结合}

SNS 作为 web 的一种运用, 其特点和功能决定了 SNS 在 E-learning 领域大有作为。国外有学者提出了 SNS 在正 式教育和非正式教育中的潜力: 话语权与建立互信; 成为 学习内容的创造者, 管理者; 作为协作与团队成员; 成为 学习探究者; 独立与达观精神; 关键的和现实世界的技能。 SNS 的互动性可以给学习者之间、学习者与教师之间、学 习者与专家之间提供更有效的交流写作空间。信息分享可 以帮助学生在获取资源的同时更好的实现知识的传播, SNS 的社会性还可以帮助学习者提高他们的应变能力和社 会认知能力。
从技术层面上来看, SNS 采用分布式技术, 营造一个 基于个体的网络基础性应用程序, 它通过客户端软件将每 个用户的个人设备、带宽资源和存储空间统筹安排, 得以 支撑起一个不依赖于中心服务器的网络平台。也就是说, 从技术层面上来讲, SNS 相当于一个开超市的地主, 它开 设一个超市, 用户购买货架来销售产品。也就是说, 用户 只要购买它的许可证 (超市的准入许可), 编写 APP (自己 准备的货架和导购人员), 就可以码货销售。在 E-learning 中, 商家担任的是组织者、策划者, 是人力资源服务商。 E-learning 商家的任务在于, 分析什么样的课程是用户需求 量最大又方便开展教学的, 并让他们有兴趣来向课程顾问 咨询; 课程顾问怎么样才能把咨询者变成学员; 策划什么 样的宣传手段让商家可提供的服务信息能迅速的在平台上 进行传播。

从应用层面上来看, SNS 让相互关联的用户平等的、 遵守同一规则的去做同一件事情。在互联网上经常做的事 情都可以算作是在接受 SNS 的应用服务, 如豆瓣、QQ、 网络游戏等。SNS 在技术和服务上都体现出一个基本理念: 平等 (黄绍麟, 2008) [6]。用户既是学习内容的接受者和 受益者, 又是学习资源的分享者和提供者。在 SNS 服务平 台上, 用户拥有同等的权利和义务: 要获取就需要有提供, 提供的越多获取的就越多。

\section{2 基于 SNS 的 E-learning 盈利模式}

目前, 国内的 E-learning 经过十几年的发展, 已经形 成了较为专业的分工和规模。现有的网络教育中, 有专门 提供师资的, 有专门招生的, 有专做教学站点的, 有专门 做服务器和提供设备租赁的, 有专门做平台或辅助软件研 发的公司, 还有专门做项目开发的顾问公司等等, 所有这 些公司或网站都围绕一个教学体系: 师生体系, 教师在网 络上授课, 学生学习。这种单一的教育体系, 已经严重制 约了 E-learning 的持续发展。构建基于 SNS 的 E-learning 创新模式, 就是要打破这种垂直单一的师生教育体系, 在 SNS 中, 不再有老师和学生的身份, 只有参与者和服务者 的身份。服务者构建各种服务区域, 参与者参与其中, 分 享各种信息和服务。

根据 SNS 的特点和功能, 结合 E-learning 现有的教育 教学模块, 基于 SNS 的 E-learning 创新商业模式将教育系 统平台分为五大区和五大模块。五大区分别为资料区、问 答区、讨论区、交易区、讲坛区。五大模块分别为在线教 学答疑模块、学员交互交流模块、学员信息模块、电子商 务交易模块、网站信息发布与管理模块。从这些模块所涉 及到的内容来看, 基于 SNS 的 E-learning 盈利模式主要来 自于五大部分：资源共享、问询解答、热门讨论、电子交 
易、设坛开讲。在资源共享方面, 参与者可以上传一些教 育资源, 自己设定下载价格, 通过服务平台赚钱, 服务商 为了刺激参与者上传高质量的资源, 甚至对上传者发放一 定奖金。在学员交流区, 参与者可以发布问题悬赏解答, 还可以对一些学科提供专家解答服务选项, 明码标价, 将 真正知识转化为 “财富”。另外, 在热门讨论区, 讨论的内 容还可以著书立说, 变成丛书或文集, 讨论者也可以以服 务的名义收取费用。至于 SNS 作为电子交易的平台, 许多 教育方面的交易在这里都可以发生, 比如资料的翻译, 书 籍的出版, 论文的发表, 甚至于一些听课证、参会证、培 训证等等都可以在这里交易。最后, 作为设坛开讲的场所, 虽然讲学和学习可以免费, 但服务商在讲坛文稿、音频、 视频等方面可以为讲学者提供很多增值服务, 从而可以从 中盈利, 另外, 讲坛完后出书、出光盘、做精品课程等等, 又可以获得二次盈利的机会。

\section{参考文献(References)}

[1] Gu Yuli, Li Dageng. Study on the development of SNS website of China: from Facebook to Xiaonei, South China Journal, 2008(7)

[2] Wan Mingyi. Discussion on the network education and continuing education, Agriculture in Gansu. 2006(4)

[3] Tian Guiping. Study on countermeasures of vigorously advancing China's network continuing education, Chinese Adult Education, 2006(4)

[4] Huang Shaolin. Study on the profit mode of SNS, Internet Weekly, 2008(7)

[5] Ding Zhe. Study on profit model of Internet, Science and Technology Intelligence, 2006(11)

[6] Cheng Shaoshan, Xi Jiasheng. Precision marketing, Beijing: Peking University press, 2006. 